\title{
Electrophysiological Monitoring of Focal and Entire Retinal Function during Treatment with Intravitreal Methotrexate for Intraocular Lymphoma
}

\author{
Takashi Matsushima Yuji Yoshikawa Airi Shimura Ayana Yajima \\ Yui Ojima Kei Shinoda \\ Department of Ophthalmology, Faculty of Medicine, Saitama Medical University, Saitama, \\ Japan
}

\section{Keywords}

Intraocular lymphoma · Intravitreal injection of methotrexate · Full-field electroretinogram · Multifocal electroretinogram

\begin{abstract}
We describe the electroretinographic findings of a case of primary intraocular lymphoma (PIOL) wherein the patient received intravitreal injections of methotrexate (ivMTX). A 62-yearold man developed blurred vision and complained of decreased visual acuity (VA) in his right eye. Fundus examination showed vitreous opacity and multiple subretinal yellowish lesions. Optical coherence tomography $(\mathrm{OCT})$ revealed subretinal and intraretinal infiltrations. The full-field electroretinogram (ffERG) showed subnormal combined rod-cone response and multifocal electroretinogram ( $\mathrm{mfERG}$ ) recorded using skin electrodes showed severe attenuation of the response compared with the other eye. Pars prana vitrectomy, phacoemulsification, and lens implantation were performed to remove the opacity, and vitreous biopsy revealed a high ratio of interleukin 10-6 (76.0). There was no systemic malignant lesion, and the patient was diagnosed with PIOL. Treatment with ivMTX $(400 \mu \mathrm{g} / 0.1 \mathrm{~mL})$ was started. One month later, the intraretinal infiltration had disappeared, and $\mathrm{mfERG}$ revealed recovery of the response density from the central area. Two months later, OCT showed recovery of the foveal ellipsoid and interdigitation zones, and VA recovered to 20/17; mfERG showed maintenance of macular function. However, the amplitude of $a$ - and b-waves in the ffERG gradually decreased. Macular function recovered, but there was also a decrease in total retinal function. mfERG and ffERG recorded using skin electrodes were useful in monitoring macular and entire retinal function with repeated examinations and showed recovery and maintenance of macular function in a case of PIOL treated with ivMTX.
\end{abstract}


Matsushima et al.: Electrophysiological Monitoring during Intravitreal Methotrexate Treatment

\section{Introduction}

An intraocular lymphoma (IOL) has several ocular symptoms, such as vitreous opacity, iritis, subretinal lesions, corneal precipitates, and retinal vasculitis [1]. Optical coherence tomography (OCT) reveals various topographic findings, such as hyperreflective subretinal infiltrates, hyperreflective infiltration in the inner retinal layers, retinal pigment epithelium (RPE) undulation, clumps of vitreous cells, and sub-RPE deposits [2]. Although these structural changes are a concern, functional assessments are still limited $[3,4]$.

A previous report demonstrated the efficiency of intravitreal injections of methotrexate (ivMTX) in treating IOL [5]. An experimental study in rabbits revealed the retinal cytotoxicity of ivMTX [6]. However, few reports have evaluated changes in retinal function after ivMTX administration in humans [3].

Conventional electroretinography (ERG) uses contact lens electrodes and is not suitable for repeated recordings. In this study, we performed ERG using skin electrodes before and after ivMTX administration.

\section{Case Presentation}

A 62-year-old man developed color vision deficiency and complained of decreased visual acuity (VA) in his right eye even though his VA was 20/20. Fundus examination showed vitreous opacity and multiple subretinal yellowish lesions. OCT revealed subretinal and intraretinal infiltrations. Fundus autofluorescence (FAF) showed multiple hyperfluorescence spots at the first visit (show in Fig. 1).

Nine months later, the vitreous opacity and infiltrations had increased and VA had decreased to 20/70. The subretinal yellowish lesion was atrophic and FAF showed hypofluorescence spots (shown in Fig. 2). A full-field ERG (ffERG) (RETeval ${ }^{\mathrm{TM}}$ system, LKC Technology Inc., Gaithersburg, MD, USA) and multifocal ERG (mfERG) were recorded in accordance with the International Society for Clinical Electrophysiology of Vision (ISCEV) standards $[7,8]$; however, both ffERG and mfERG were recorded using skin electrodes (LE4100; Mayo Corporation, Inazawa, Japan). Great care was taken to ensure appropriate fixation during mfERG recordings. Briefly, crosses were presented as fixation targets on the central stimulus element and the patient was instructed to look at the crosses during recordings. In addition, the examiner carefully observed and assessed the stability of fixation at each
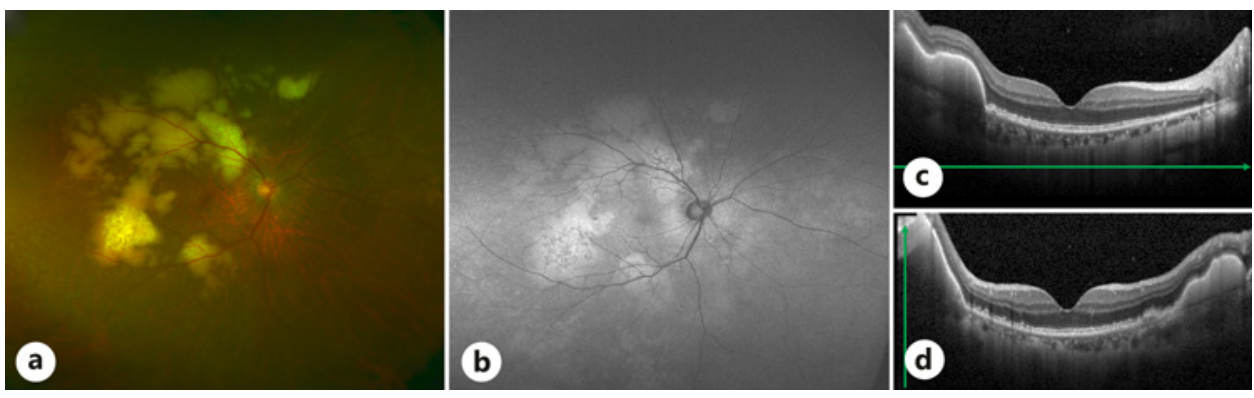

Fig. 1. a Fundus photograph showing subretinal yellowish lesions. b FAF showing hyperfluorescence lesions corresponding to the subretinal yellowish lesions. OCT showing a massive sub-RPE lesion and pre-RPE deposits (horizontal scan (c); vertical scan (d)). FAF, fundus autofluorescence; OCT, optical coherence tomography; RPE, retinal pigment epithelium. 
Matsushima et al.: Electrophysiological Monitoring during Intravitreal Methotrexate Treatment
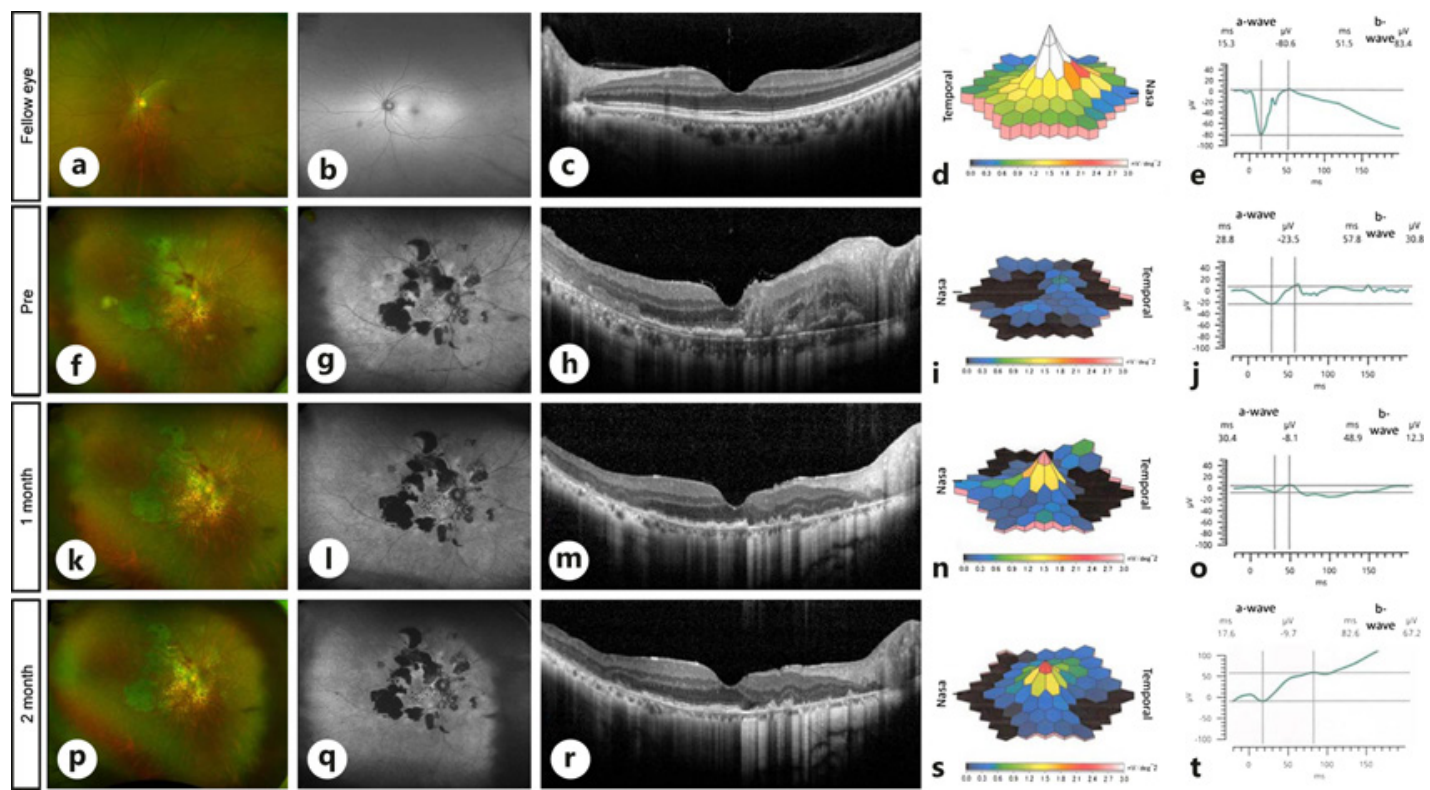

Fig. 2. Ophthalmologic examination of the unaffected eye (a-e), and the clinical course of PIOL treatment with intravitreal methotrexate (f-t). $\mathbf{f}$ Twelve months after the first visit, the subretinal yellowish lesion changed to an atrophic lesion and retinal hemorrhage occurred. $\mathbf{g}$ FAF showing change of the hyperfluorescence lesion to a hypofluorescence lesion. $\mathbf{h}$ OCT showing intraretinal infiltration, sub-RPE deposits, and disruption of the ellipsoid zone. mfERG showing severe attenuation of response density (i) and ffERG showing subnormal change compared with the unaffected eye $(\mathbf{e}, \mathbf{j})$. After treatment with ivMTX, retinal hemorrhage gradually disappeared (k, p). However, the hypofluorescence lesion did not change during the 2 months after starting treatment $(\mathbf{l}, \mathbf{q})$. OCT showed disappearance of intraretinal infiltration and recovery of the foveal ellipsoid and interdigitation zone ( $\mathbf{m}, \mathbf{r})$. At the same time, mfERG showing recovery of central response density $(\mathbf{n}, \mathbf{s})$. However, the response of ffERG gradually decreased after treatment $(\mathbf{o}, \mathbf{t})$. PIOL, primary intraocular lymphoma; FAF, fundus autofluorescence; RPE, retinal pigment epithelium; OCT, optical coherence tomography; mfERG, multifocal electroretinogram; ffERG, full-field electroretinogram; ivMTX, intravitreal methotrexate.

recording. The ffERG showed subnormal combined rod-cone response, and the mfERG showed severe attenuation of the response density in the central area as compared with the other eye (shown in Fig. 2; online suppl. Fig. 1, 2; for all online suppl. material, see www. karger.com/doi/10.1159/000514189). Pars prana vitrectomy, phacoemulsification, and lens implantation were performed to remove the opacity. A vitreous biopsy was also performed. The ratio of interleukin 10-6 was 76.0, and vitreous cytology showed class IIIb. There was no systemic malignant lesion and a diagnosis of primary intraocular lymphoma (PIOL) was made. The FAF and OCT showed disseminated areas of RPE atrophy, a disorganized outer retinal layer, and macular edema (shown in Fig. 2). ivMTX ( $400 \mu \mathrm{g} / 0.1 \mathrm{~mL}$ ) was administered once a week for 2 months as a loading dose and then once a month for 12 months as a maintenance dose. Over the month following the first dose of ivMTX, the subretinal yellowish lesion and intraretinal infiltration gradually disappeared (shown in Fig. 2). The mfERG showed that the implicit time of $\mathrm{N} 1$ and $\mathrm{P} 1$ recovered to the same level as in the unaffected eye in the central $5^{\circ}$ and $20^{\circ}$ areas, but the amplitude of $\mathrm{N} 1$ and P1 did not reach these levels. Two months later, OCT showed recovery of the foveal ellipsoid and interdigitation zones. VA recovered to $20 / 17$ and the mfERG showed maintenance of macular function. However, the amplitude of a- and b-waves on ffERG gradually decreased (shown in Fig. 2, 3). 
Matsushima et al.: Electrophysiological Monitoring during Intravitreal Methotrexate Treatment
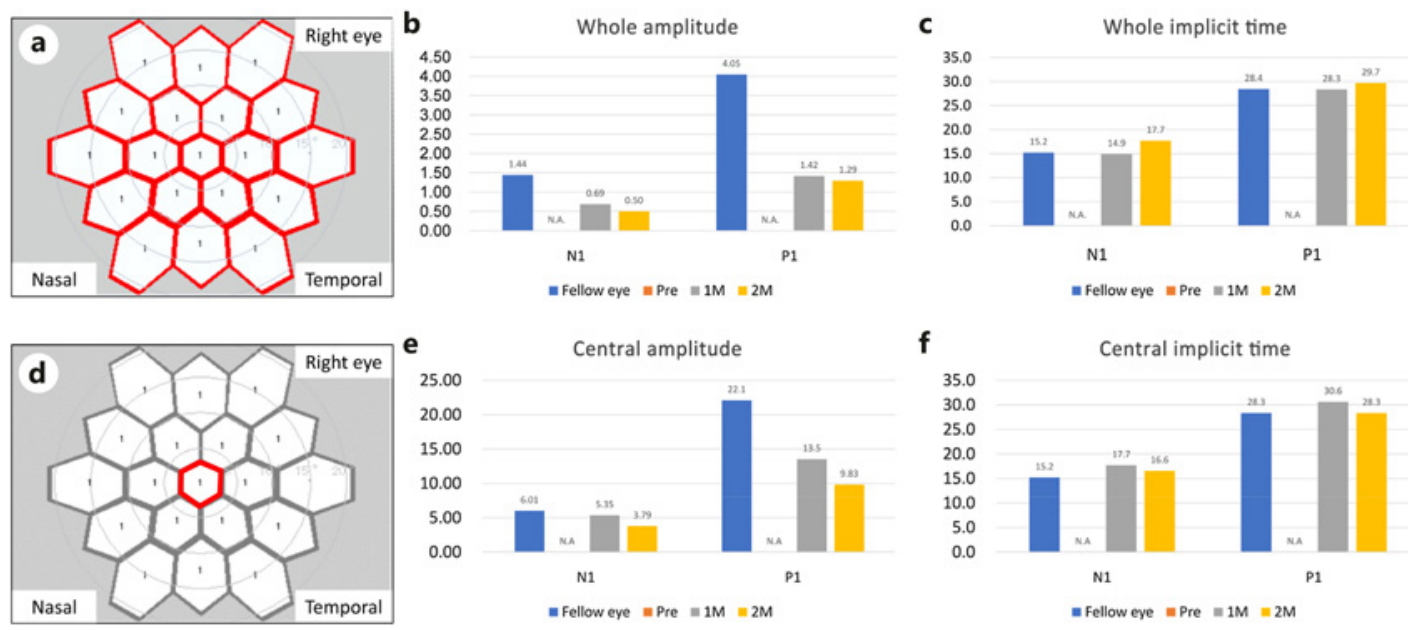

Fig. 3. mfERG recorded with skin electrodes at the center of the fovea covering a visual field of $30^{\circ}$. A response density of the whole area (a) and central area (d) were analyzed to compare the amplitude and implicit time before (Pre) and after treatment ( 1 and 2 months after treatments) (b, c, e, f). The implicit time of $\mathrm{N} 1$ and P1 recovered to levels as were seen for the unaffected eye $(\mathbf{c}, \mathbf{f})$. The amplitude of N1 and P1 did not reach the values as were seen for the unaffected eye (b, e). mfERG, multifocal electroretinogram.

\section{Discussion and Conclusion}

ivMTX is an effective treatment for IOL. However, few reports of functional assessment after ivMTX have been published $[3,9]$. Repeated injections are considered feasible without retinotoxicity [10], and OCT studies reveal structural recovery after treatment with ivMTX [11]. However, an experimental study in rabbits analyzed retinal function by ERG after ivMTX, showing significant reduction of the amplitude of a- and b-waves [6]. These results suggest that there are possible subclinical complications of ivMTX which can only be assessed by ERG.

A previous case report described unilateral PIOL treated with ivMTX and prominent suppression of the central signal compared with that in the unaffected eye in mfERG [9]. Another case report showed improvement on ffERG after systemic chemotherapy. The authors reported that the negative pattern disappeared, but the amplitude still did not reach normal ranges [4]. Therefore, to evaluate the ophthalmologic retinal efficiency and safety of ivMTX and/or systemic chemotherapy for eyes with IOL, repeated functional examinations should be performed during the course of treatment.

Although ERG examination is useful to determine the physiological status of the retina, it is not routinely performed. One reason is that it requires the use of contact lens electrodes, which can cause corneal abrasions and infections [12]. We believe that ERG examination using skin electrodes can be performed repeatedly because it can reduce the risk of corneal abrasion and infection. It can also be more comfortable for the patient.

In the present case, mfERG and ffERG recordings with skin electrodes were used to examine changes in the macular as well as entire retinal function before and after ivMTX. A previous study of age-related macular degeneration reported that intravitreal aflibercept recovered responses on focal macular ERG; however, the response on ffERG gradually decreased [13]. Similar to the findings of a previous report [9], there was severe attenuation of the response density from the central area before treatment with ivMTX as seen using mfERG (shown in Fig. 2,3) in the present case. However, recovery was confirmed on mfERG 1 month after weekly ivMTX administration. Moreover, the central response density was maintained and achieved excellent VA at 2 months post-treatment. It is deemed that the change in mfERG reflects functional recovery due to the resolution of macular edema followed by ivMTX treatment.

\section{Karger'}


In contrast, Egawa et al. [3] reported a case of PIOL treated with ivMTX and mfERG which did not show recovery despite the recovery of retinal structures. One explanation is that this previous case involved extensive disorganization of the outer retinal layer even after treatment, whereas the present case involved outer retinal layer recovery in relatively wide areas. In a previously reported case by Casady et al. [14], there was a diffuse hypofluorescence spot in the macular area, which was more severe than in the present case, suggesting the presence of diffuse and irreversible retinal damage. However, both the current case and previous case showed recovery of the foveal ellipsoid and interdigitation zones and excellent VA after ivMTX.

The fellow eye had no symptoms, and the fundus and OCT examinations revealed normal findings with a b/a wave ratio of approximately 1.0. But the ffERG showed a relatively small b-wave with a normal a-wave. Yasuda et al. [4] reported that in a patient with IOL, the amplitude of the b-waves was distinctly reduced and was smaller than the a-waves, resulting in the "negative type" ERG for both eyes. After chemotherapy, there was quick improvement in relation to the b-wave and no "negative type" ERG was observed for both eyes. However, in the convalescent stage, when the ocular manifestations were resolved, complete recovery was not observed on both ffERG and mfERG. Although the ffERG of the left eye in the present case was not a "negative type" ERG, it may imply subclinical change. Careful observation with repeated structural and functional assessments is warranted.

There are several limitations in this report. First, we did not perform retinal biopsy and histological examination. However, we considered this case as PIOL based on the high IL $10 / 6$ ratio and class IIIb by cytology, which are useful in the diagnosis of IOL [15]. Second, poor fixation stemming from poor VA before treatment might play a role in the severely attenuated central response seen on the mfERG. However, we believe the results are reliable because a stable fixation was ensured by the following: the patient had a VA of 20/70; there was no central scotoma, which was confirmed using perimetry (shown in online suppl. Fig. 2); fixation targets were presented on the display; and the fixation was carefully observed. Third, although mfERG showed recovery of central retinal response density after ivMTX, we could not deny the retinal toxicity of ivMTX. The ffERG showed attenuation of a- and b-wave amplitude that may reflect diffuse retinal toxicity. However, improvement of ultrastructure and FAF suggest at least the therapeutic effect of ivMTX and support improvement of response on mfERG. The ffERG may be useful in evaluating the effects of ivMTX on the extensive retinal area. Long-term observation was required to assess retinal complications and functional change following ivMTX. Furthermore, separate evaluation of both macular and entire retinal function may be important for the assessment of retinal toxicity. mfERG and ffERG recorded using skin electrodes could monitor macular and entire retinal function with repeated examinations and were used to assess the recovery and maintenance of macular and entire retinal function in the present case of PIOL treated with ivMTX.

\section{Acknowledgement}

The authors thank Editage (https://www.editage.jp) for the English language review.

\section{Statement of Ethics}

This study was approved by the Ethics Committee of Saitama Medical University. Written informed consent was obtained from the patient in this case report and for publication of this article and the accompanying images. A copy of the written consent is available for review by the editor of this journal.

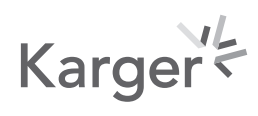


Matsushima et al.: Electrophysiological Monitoring during Intravitreal Methotrexate Treatment

\section{Conflict of Interest Statement}

The authors have no conflicts of interest to declare.

\section{Funding Sources}

This study was supported in part by the Japan Society for the Promotion of Science (JSPS; KAKENHI grant number: 17K11430).

\section{Author Contributions}

Y.Y. cared for the patient, performed the workups, and carried out the treatment. T.M., A.S., A.Y., Y.O., and Y.Y. prepared the figures and collected data, analyzed the ophthalmological findings, and gave critical suggestions. Y.Y. and K.S. prepared the manuscript. All authors agree to be accountable for all aspects of work. Patient anonymity is preserved. All authors attest that they meet the current ICMJE criteria for authorship.

\section{References}

1 Kimura K, Usui Y, Goto H; Japanese Intraocular Lymphoma Study G. Clinical features and diagnostic significance of the intraocular fluid of 217 patients with intraocular lymphoma. Jpn J Ophthalmol. 2012 Jul;56(4): 383-9.

2 Barry RJ, Tasiopoulou A, Murray PI, Patel PJ, Sagoo MS, Denniston AK, et al. Characteristic optical coherence tomography findings in patients with primary vitreoretinal lymphoma: a novel aid to early diagnosis. $\mathrm{Br} \mathrm{J}$ Ophthalmol. 2018 Oct;102(10):1362-6.

3 Egawa M, Mitamura Y, Hayashi Y, Semba K, Naito T. Changes of fundus autofluorescence and spectral-domain optical coherence tomographic findings after treatment of primary intraocular lymphoma. J Ophthalmic Inflamm Infect. 2014 Dec;4(1):7.

4 Yasuda C, Ueno S, Kondo M, Kondo N, Piao CH, Terasaki H. Analyses of ERG in a patient with intraocular lymphoma. Clin Ophthalmol. 2010;4:301-6.

5 de Smet MD, Vancs VS, Kohler D, Solomon D, Chan CC. Intravitreal chemotherapy for the treatment of recurrent intraocular lymphoma. Br J Ophthalmol. 1999 Apr;83(4):448-51.

6 Aly E, Ebrahim A. Apoptosis and electroretinogram after intravitreal injection of methotrexate in an experimental rabbit model. Gen Physiol Biophys. 2016 Apr;35(2):231-6.

7 Hood DC, Bach M, Brigell M, Keating D, Kondo M, Lyons JS, et al. ISCEV standard for clinical multifocal electroretinography (2011 edition). Doc Ophthalmol. 2012 Feb;124(1):1-13.

8 McCulloch DL, Marmor MF, Brigell MG, Hamilton R, Holder GE, Tzekov R, et al. ISCEV standard for full-field clinical electroretinography (2015 update). Doc Ophthalmol. 2015 Feb;130(1):1-12.

9 Kim E, Kim C, Lee J, Cho Y. A case of primary intraocular lymphoma treated by intravitreal methotrexate. Korean J Ophthalmol. 2009 Sep;23(3):210-4.

10 Velez G, Yuan P, Sung C, Tansey G, Reed GF, Chan CC, et al. Pharmacokinetics and toxicity of intravitreal chemotherapy for primary intraocular lymphoma. Arch Ophthalmol. 2001 Oct;119(10):1518-24.

11 Zhao H, Wang X, Mao Y, Peng X. Longitudinal observation of OCT imaging is a valuable tool to monitor primary vitreoretinal lymphoma treated with intravitreal injections of methotrexate. BMC Ophthalmol. 2020 Dec; 20(1): 10 .

12 Coupland SG. Electrodes for visual testing. In: Heckenlively JR, Arden GB, editors. Principles and practice of clinical electrophysiology of vision. Cambridge, MA: MIT Press; 2006. p. 245-54.

13 Nishimura T, Machida S, Hara Y. Changes in cone-driven functions after intravitreal aflibercept injections in patients with age-related macular degeneration. Doc Ophthalmol. 2020 Oct;141(2):137-47.

14 Casady M, Faia L, Nazemzadeh M, Nussenblatt R, Chan CC, Sen HN. Fundus autofluorescence patterns in primary intraocular lymphoma. Retina. 2014 Feb;34(2):366-72.

15 Chan CC, Whitcup SM, Solomon D, Nussenblatt RB. Interleukin-10 in the vitreous of patients with primary intraocular lymphoma. Am J Ophthalmol. 1995 Nov;120(5):671-3. 